\title{
Quilotórax bilateral após esvaziamento cervical: um raro relato de caso
}

\author{
Bilateral chylothorax after neck dissection: a rare case report
}

Quilotórax bilateral después de la disección del cuello: reporte de um caso raro

Cisa Adrine Silva Salgado ${ }^{1 *}$, Larissa Pessoa de Oliveira², Felipe Jezini III¹.

\begin{abstract}
RESUMO
Objetivo: relatar um caso de quilotórax bilateral após esvaziamento cervical devido a carcinoma de língua, complicação rara descrita 65 vezes na literatura. Detalhamento do caso: mulher, 63 anos, atendida no ambulatório de cirurgia de cabeça e pescoço com grande lesão vegetante em borda lateral esquerda da língua, a biopsia incisional diagnosticou carcinoma espinocelular, paciente então foi encaminhada para realizar glossectomia parcial à esquerda associado a esvaziamento cervical radical tipo 2 à esquerda e esvaziamento cervical supra homoióideo à direita. Evoluiu com dispneia moderada no $2^{\circ}$ dia pós operatório, através do exame físico, radiografia de tórax e análise do líquido pós toracocentese foi diagnosticado quilotórax bilateral, uma complicação extremamente rara, uma vez que não houve lesão do ducto torácico. Considerações finais: trata-se de uma complicação rara e importante, o melhor método para minimizar as consequências e a taxa de morbidade é o diagnóstico precoce. Logo, deve-se aprimorar os registros na literatura e o manejo clínico cirúrgico e clínico a fim de obter prognóstico melhor desses pacientes.
\end{abstract}

Palavras-chave: Quilotórax, Neoplasias de cabeça e pescoço, Oncologia cirúrgica.

\section{ABSTRACT}

Objective: to report a case of bilateral chylothorax after neck dissection due to tongue carcinoma, a rare complication described 65 times in the literature. Case Details: a 63-year-old woman attended at the head and neck surgery clinic with large vegetating lesion on the left lateral edge of the tongue, the incisional biopsy diagnosed squamous cell carcinoma, the patient was then referred for partial left glossectomy associated with radical neck dissection 2 on the left and supra-homoid neck dissection on the right. She evolved with moderate dyspnea on the 2nd postoperative day, through physical examination, chest X-ray and post-thoracentesis fluid analysis then bilateral chylothorax was diagnosed, an extremely rare complication, since there was no injury to the thoracic duct. Final considerations: this is a rare and important complication, the best method to minimize the consequences and the rate of morbidity is early diagnosis. Therefore, the records in the literature and clinical, surgical and clinical management should be improved in order to obtain a better prognosis for these patients.

Keywords: Chylothorax, Head and neck neoplasms, Surgical oncology.

\section{RESUMEN}

Objetivo: reportar un caso de quilotórax bilateral después de una disección del cuello por carcinoma de lengua, una complicación rara descrita 65 veces en la literatura. Detalles del caso: una mujer de 63 años atendida en la clínica de cirugía de cabeza y cuello con gran lesión vegetativa en el lado izquierdo de la lengua, la biopsia incisional diagnosticada carcinoma de células escamosas, el paciente fue derivado para una glossectomía parcial izquierda asociada con disección radical del cuello 2 a la izquierda y disección del cuello supra-homoide a la derecha. Evolucionó con disnea moderada en el segundo día postoperatorio, mediante examen físico, radiografía de tórax y análisis de líquido post-toracocentesis, se diagnosticó quilotórax bilateral, una complicación extremadamente rara, ya que no hubo lesión en el conducto torácico. Consideraciones finales: Es una complicación rara e importante, el mejor método para minimizar las consecuencias y la tasa de morbilidad es el diagnóstico precoz. Por lo tanto, los registros en la literatura y el manejo clínico, quirúrgico y clínico deben mejorarse para obtener un mejor pronóstico para estos pacientes.

Palabras clave: Quilotórax, Neoplasias de cabeza y cuello, Oncología quirúrgica.

\footnotetext{
${ }^{1}$ Fundação Centro de Controle de Oncologia do Estado do Amazonas (FCECON), Manaus - AM.

*E-mail: cisa_adrine@hotmail.com

2 Universidade Federal do Amazonas (UFAM), Manaus - AM.
}

ACEITO EM: $8 / 2020$

PUBLICADO EM: 10/2020 


\section{INTRODUÇÃO}

O câncer da cavidade oral é definido como neoplasia maligna que pode afetar lábios, gengivas, palato e língua. Segundo o Instituto Nacional de Câncer (INCA), estima-se que até o final de 2020 serão 15.190 casos no Brasil (INSTITUTO NACIONAL DE CÂNCER (INCA), 2019). Esse tipo de tumor é mais prevalente em pacientes do sexo masculino durante a quinta e sexta década de vida, e tem como principais fatores de risco o tabagismo, etilismo e infecções orais pelo HPV, principalmente os subtipos 16, 18, 31 e 33 (DEDIVITIS RA, et al., 2004; SILVA CC, et al., 2010).

As lesões neoplásicas de língua constituem um tipo de câncer de caráter agressivo, com evolução insidiosa, é sítio mais acometido dos carcinomas orais, representando até $50 \%$ dos casos desse tipo de neoplasia (DEDIVITIS RA, et al., 2004).

Clinicamente tem diversas formas de apresentação, no início se manifesta como lesão ulcerada associada a poucos sintomas, à medida que a patologia progride é possível observar diversas outras manifestações como massas exofíticas ou endofíticas de aspecto leucoplásico ou eritroplásico, geralmente associadas a dor, sangramentos, odinofagia, disfagia e tumefação cervical (SILVA CC, et al., 2010).

O tipo histológico mais comum é o carcinoma espinocelular também denominado carcinoma de células escamosas, representando $90 \%$ dos casos, seguido pelo carcinoma verrucoso, com $5 \%$ dos casos. Apresenta elevadas taxas de metástases linfonodais cervicais de forma precoce, devido à rica drenagem linfática da região, de 60 a $90 \%$ de forma ipsilateral e de 10 a $20 \%$ bilateralmente, sendo o prognóstico inversamente proporcional à quantidade de linfonodos acometidos por metástase. Outros fatores de pior prognóstico são o acometimento vascular e nervoso, além da invasão da linha média e a presença de neoangiogênese (RAPOPORT A, et al, 2006).

Um dos tratamentos é a ressecção cirúrgica da lesão primária com margens de segurança associada a linfadenectomia cervical também denominada de esvaziamento cervical (KOHLER HF, et al, 2010). A cirurgia consiste na remoção em bloco dos grupos de linfonodos e vasos linfáticos que compõem as principais redes de drenagem, e pode ou não envolver a retirada de estruturas adjacentes como o músculo esternocleidomastoideo, veia jugular interna e nervo acessório (DEDIVITIS RA, et al., 2011).

É um procedimento essencial, uma vez que auxilia no estadiamento da doença, estabelece a necessidade de tratamento complementar, como rádio ou quimioterapia e trata a disseminação linfática. As taxas de complicações intra e pós-operatórias ainda são muito variáveis, entre as principais é possível citar hemorragia, embolia gasosa, edema facial, fistula quilosa e lesão de estruturas nervosas como o nervo vago e o nervo acessório, que estão entre as mais comuns (DEDIVITIS RA, et al., 2011).

Uma complicação rara desse procedimento descrita na literatura é o quilotorax sendo o acometimento bilateral observado em apenas 65 casos até o momento. Ocorre o acúmulo de linfa no espaço pleural e sucede geralmente devido a vazamentos externos de linfa, lesão ou obstrução do ducto torácico.

No entanto, tais etiologias não foram observadas nas cirurgias cervicais relatadas, sendo a fisiopatologia dessa complicação ainda desconhecida, logo, observa-se a necessidade do registro e investigação desses casos.

O quilotórax é uma complicação comum em cirurgias de cabeça e pescoço que abordam a região cervical esquerda, porém a apresentação bilateral é extremamente rara, representando 1-2\% dos casos (RUNGE T, et al., 2014; SINGH R, et al, 2006).

O objetivo desse estudo foi relatar um caso de quilotórax bilateral após esvaziamento cervical em uma paciente com diagnóstico de carcinoma espinocelular em borda lateral esquerda de língua e discutir fatores que levam a essa complicação, além da importância do manejo precoce identificado em um Hospital referência em Oncologia da cidade de Manaus. Trata-se de uma complicação extremamente rara principalmente em cirurgia de cabeça e pescoço. 


\section{DETALHAMENTO DO CASO}

Paciente S.F.O, sexo feminino, 63 anos, parda, natural e procedente de Manaus, Amazonas, procurou o sistema de saúde devido a lesão ulcerada dolorosa na borda lateral esquerda da língua com evolução de 6 meses. Ao exame físico, observou-se lesão vegetante, com bordas irregulares (Figura 1), e linfonodos palpáveis em níveis IB, II e III da região cervical à esquerda, todos menores que $6 \mathrm{~cm}$, paciente com estadiamento clínico T3N2Bm0.

Ex-tabagista em abstinência há 2 anos. Sem história familiar de neoplasia. Hipertensa em uso de losartana e anlodipino. Realizada biopsia incisional em abril de 2019 diagnosticou-se carcinoma de células escamosas moderadamente diferenciado. Paciente então foi encaminhada para realizar tratamento padronizado com glossectomia parcial à esquerda associado a esvaziamento cervical radical modificado tipo 2 à esquerda (ressecção de veia jugular interna ipsilateral) e esvaziamento cervical supraomohioideo à direita.

Paciente ficou internada na enfermaria em regular estado geral, traqueostomizada, com drenos suctores em região cervical direita e esquerda, ambos com débito de aspecto sanguinolento, aceitando dieta por sonda nasoenteral, no $2^{\circ}$ dia pós-operatório (DPO) evoluiu com lombalgia, ao exame físico, a ausculta torácica evidenciou murmúrio vesicular sem ruídos adventícios diminuído bilateralmente.

Paciente então realizou radiografia (Figura 2) e tomografia computadorizada de tórax (Figura 3) para investigação diagnóstica, os quais revelaram derrame pleural bilateral de volume moderado, realizada toracocentese bilateral com drenagem $1000 \mathrm{ml}$ (Figura 4) de líquido pleural de aspecto purulento do lado esquerdo $300 \mathrm{ml}$ do lado direito com as mesmas características, logo após, realizada drenagem fechada de tórax em selo d'água bilateralmente. A análise bioquímica evidenciou $788 \mathrm{mg} / \mathrm{dL}$ de triglicerídeos, sem crescimento bacteriano ou fúngico, confirmando o diagnóstico de quilotórax.

A equipe médica assistente optou pela reabordagem cirúrgica, realizado cervicotomia exploradora sem evidências de extravasamentos nas ligaduras do ducto torácico e ducto linfático direito. $O$ tratamento foi realizado de forma conservadora com antibioticoterapia (Meropenem e Vancomicina), nutrição mista (nutrição enteral hipolipídica e nutrição parenteral) e correção dos distúrbios hidroeletrolíticos.

Após uma semana paciente apresentou diminuição considerável da drenagem quilosa, os drenos em selo d'água foram retirados após 15 dias de tratamento e paciente recebeu alta hospitalar com os devidos acompanhamentos e seguimento ambulatorial.

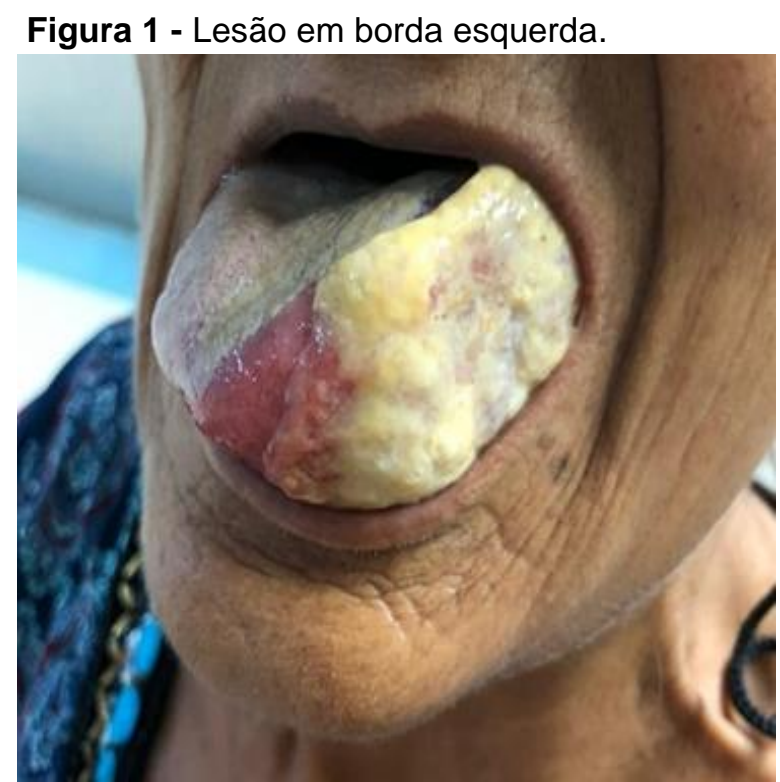

Fonte: Salgado CAS, et al., 2020. 
Figura 2 - Radiografia de tórax no 2ํㅡㄹ.

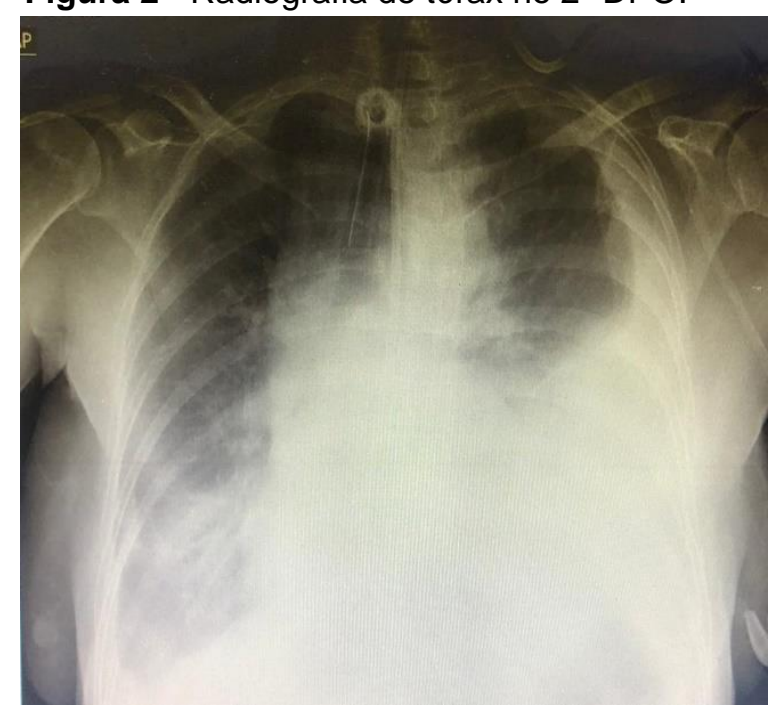

Fonte: Salgado CAS, et al., 2020.

Figura 3 - TC evidenciando derrame pleural bilateral.

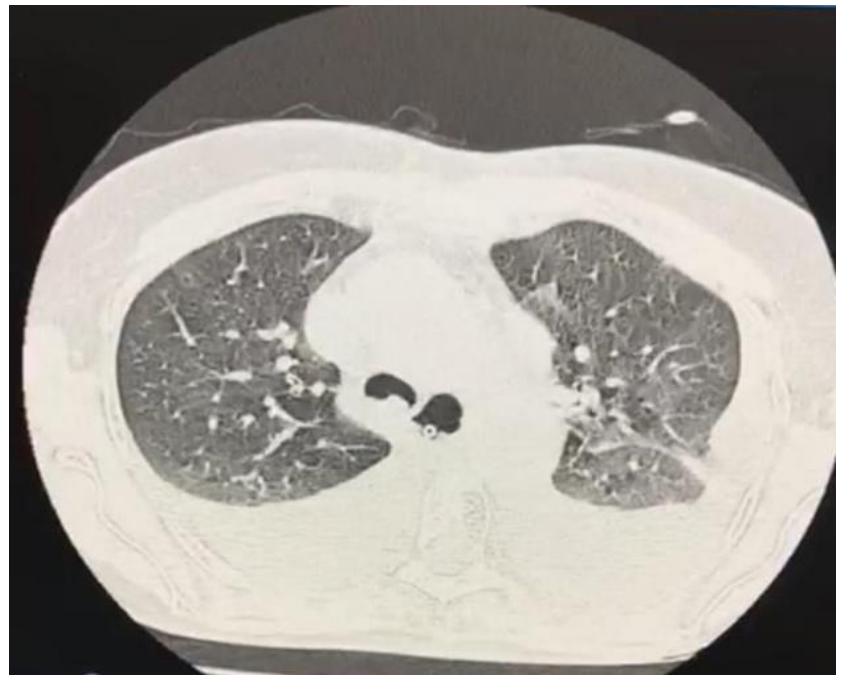

Fonte: Salgado CAS, et al., 2020.

Figura 4 - Líquido pleural pós toracocentese.

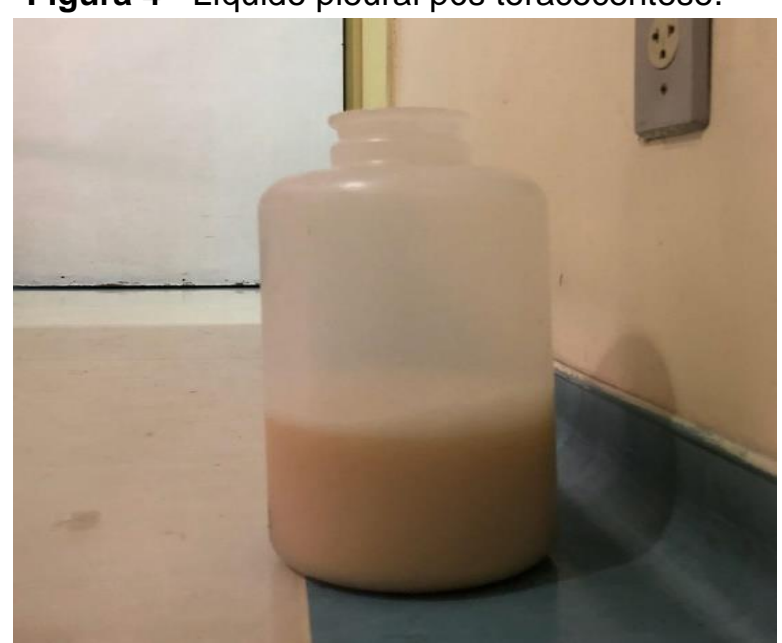

Fonte: Salgado CAS, et al., 2020. 
Realizada a biopsia do material pós-cirúrgico, foi evidenciado carcinoma de células escamosas ceratinizante moderadamente diferenciado com infiltração da musculatura, glândulas salivares adjacentes, perineural e vascular. Além disso, foi possível observar carcinoma de células escamosas metastático para 10 linfonodos de 52 nódulos isolados da peça operatória. Dessa forma, foi estabelecido estadiamento patológico de Pt3Pn2c. Paciente foi encaminhada para seguimento clínico ambulatorial.

\section{DISCUSSÃO}

Apresentamos um caso de quilotórax bilateral pós esvaziamento cervical devido a carcinoma espinocelular de língua em um hospital de referência em oncologia do Amazonas, na cidade de Manaus. O carcinoma espinocelular de língua representa $50 \%$ dos casos de neoplasias da cavidade oral, geralmente acomete 0 bordo lateral posterior e a face ventral do órgão (SILVA CC, et al, 2010).

É mais frequente em homens entre 50 e 60 anos, sendo o tabagismo o principal fator de risco. Em nosso caso, nota-se que a paciente era do sexo feminino, porém dentro da faixa etária de risco e apresentando o tabagismo como principal fator predisponente, reafirmando os estudos descritos na literatura (DEDIVITIS RA, et al., 2010).

Inicialmente tem caráter clínico sutil, podendo apresentar sinais e sintomas em fases mais avançadas da doença, logo faz-se necessária observação atenta dos profissionais de saúde ao menor sinal de lesão na cavidade oral. Apresenta altas taxas de metástase devido ao elevado número de vasos linfáticos na região cervical. $O$ tratamento pode ser por quimioterapia, radioterapia e cirurgia para ressecção da lesão em conjunto com esvaziamento cervical, o procedimento pode apresentar complicações intra e pós operatórias entre as principais é possível citar hemorragia, lesão nervosa, edema, fístulas, entre outras (SRIKUMAR S, et al., 2006).

As fistulas quilosas são raras, representando $1-2 \%$ dos casos, sendo que a maioria ocorre do lado esquerdo. Outra complicação rara é o quilotorax, pode ser uni ou bilateral, até o momento foram descritos apenas 65 casos na literatura (SINGH R, et al., 2016; SRIKUMAR S, et al., 2006). Pode ser definido como acúmulo de linfa no espaço pleural, e de acordo com a etiologia pode ser classificado como espontâneo, obstrutivo ou traumático.

A causa mais comum é a traumática por acidentes ou devido a lesões iatrogênicas do ducto torácico durante procedimentos cirúrgicos. Obstruções extrínsecas ou intrínsecas também são causas comuns, principalmente na presença de neoplasias. Por último, quilotorax espontâneo é uma condição rara, geralmente associado a condições congênitas (BUSQUETS JM, et al., 2004).

Inicialmente é assintomático e tem caráter insidioso, à medida que progride, o paciente pode apresentar dispneia, taquipneia, diminuição do murmúrio vesicular, hipotensão entre outros sintomas, podendo evoluir para insuficiência respiratória (RODRIGUES ALL, et al., 2012).

O complemento diagnóstico pode ser feito por exames de imagem como radiografia ou tomografia de tórax, e análise do líquido pós drenagem. O manejo clínico costuma ser conservador, com restrições alimentares, drenagem pleural em selo d'água e auxílio farmacológico, além de fisioterapia respiratória, tais medidas tem como objetivo reduzir a produção de quilo e minimizar a perda ponderal e de eletrólitos (XARÁ S, et al., 2013).

Caso seja observado drenagem de quilo acima de 1 litro por dia está indicado o tratamento cirúrgico com manejo direto do ducto torácico em casos de lesão ou obstrução através de cirurgia aberta ou toracoscópica por vídeo. Já nos casos em que o ducto se encontra intacto pode ser necessária uma derivação pleuroperitoneal ou pleurovenosa como último recurso (BENDER B, et al., 2016).

Um tratamento clínico inovador propõe o uso de octreodito, é um análogo sintético da somatostatina e tem a capacidade de inibir a secreção de hormônios digestivos como glucagon, líquido pancreático, bile, entre outro. Portanto, essa droga tende a diminuir o fluxo sanguíneo e linfático para órgãos internos, além de gerar 
contração dos músculos lisos, por conseguinte diminui a taxa de fluxo do ducto torácico. Artigos recentes mostram melhora do quilotórax em 2 - 3 dias após administração de octrotido, no entanto, trata-se de pesquisa recente e ainda não há consenso sobre o uso desse medicamento no manejo de quilotórax pós cirúrgico (HAYASHIBARA N, et al., 2016).

O mecanismo fisiopatológico dessa complicação pós esvaziamento cervical é pouco conhecido, duas teorias foram propostas, a primeira propõe o extravasamento direto de linfa do sítio cervical para as redes linfáticas no mediastino devido a lesão direta na porção cervical do ducto torácico. Tal estrutura anatomicamente tem origem no abdômen e drena o lado esquerdo do corpo próximo à quinta e sexta vértebra torácica.

Posteriormente, ascende à área cervical e realiza drenagem linfática na região da junção da veia subclávia esquerda e as veias jugulares internas, no entanto pode apresentar diversas variações anatômicas principalmente na porção terminal, o ducto pode ter fim na veia jugular externa, veia vertebral, veia cervical transversal, veia braquiocefálica ou veia supraescapular. Logo, é uma estrutura manipulada nas cirurgias de cabeça e pescoço (SHARMA, et al., 2018). Em nosso caso, porém, não houve lesão do ducto torácico no intraoperatório, evidenciado na reabordagem cirúrgica, ressaltando a raridade do caso apresentado.

A segunda hipótese supõe que o quiltórax pode ocorrer devido ao aumento da pressão intraluminal no ducto torácico após a ligadura desse na cirurgia acompanhado de pressão negativa intratorácica, o que pode resultar em danos na região torácica dessa estrutura (BAKASHI J, et al., 2004; SHARMA, et al., 2018).

O quilotórax leva à perda de eletrólitos, proteínas e de material orgânico lipossolúvel, fatores que resultam em distúrbios metabólicos, nutricionais e podem propiciar infecções. Além disso, o grande volume acumulado no espaço pleural tende a resultar em comprometimento cardiorrespiratório, aumentando a morbimortalidade no pós-operatório (SHARMA AK, et al., 2018; SUH S, et al., 2017).

\section{CONSIDERAÇÕES FINAIS}

Após revisão bibliográfica foi possível concluir que o quilotórax bilateral após esvaziamento cervical é uma complicação extremamente rara e importante, pode apresentar evolução clínica rápida, logo a equipe multidisciplinar de cirurgia de cabeça e pescoço deve analisar tal diagnóstico principalmente quando o paciente evolui com dispneia e derrame pleural no pós-operatório. É essencial o diagnóstico precoce e aprimoramento do suporte clínico tanto no intra quanto no pós operatório a fim de evitar complicações e reduzir a taxa de mortalidade. Trazendo a nossa região, o Amazonas possui grande extensão territorial e poucos centros de referência em cirurgia de cabeça e pescoço, o que dificulta a mobilidade e o diagnóstico destes pacientes em casos de queixas menores.

\section{REFERÊNCIAS}

1. Instituto Nacional de Câncer José Alencar Gomes da Silva. Estimativa 2020: incidência de câncer no Brasil / Instituto Nacional de Câncer José Alencar Gomes da Silva. - Rio de Janeiro: INCA, 2019.

2. DEDIVITIS RA, et al. Características clínico-epidemiológicas no carcinoma espinocelular de boca e orofaringe. Revista Brasileira de Otorrinolaringologia, 2004;70(1):35-40

3. SILVA CC, et al. Carcinoma espinocelular da língua-factores de risco e importância do reconhecimento de lesões pré-malignas. Revista Portuguesa de Estomatologia, Medicina Dentária e Cirurgia Maxilofacial, 2010;51(1):49-55.

4. RAPOPORT A, et al. Paradigma da disseminação linfática no carcinoma. Revista do Colégio Brasileiro dos Cirurgiões, 2006;33 (2).

5. KOHLER HF, et al. Impact of modified radical neck dissections on the number of retrieved nodes, recurrence and survival. Brazilian Journal of Otorhinolaryngology, 2010;76(3):374-7.

6. DEDIVITIS RA et al. Neck dissection complications. Brazilian Journal of Otorhinolaryngology, 2011;77(1):65-9

7. RUNGE T, et al. Bilateral chylothorax following neck dissection: A case report. BMC Research Notes. 2014;7(1):1-2.

8. SINGH R, et al. Bilateral Chylothorax Following Neck Dissection: Case Report \& Review of Literature. Indian Journal of Surgical Oncology, 2016;7(1):115-118.

9. SRIKUMAR S, et al. Bilateral chylothorax following left-sided radical neck dissection. The Journal of Laryngology \& Otology, 2006;120(8):705-7. 
10. BAKASHI J, et al. Bilateral chylothorax after neck dissection- A sporadic case with new treatment modality. Otorhinolaryngology Neck Surgery, 2019;4(1):1-3

11. BUSQUETS JM, et al. Bilateral chylothorax after neck dissection. Otolaryngology-Head and Neck Surgery, 2004;130(4):492-5.

12. RODRIGUES ALL, et al. Quilotórax bilateral espontâneo após vômitos excessivos em criança. Revista Paulista de Pediatria, 2016;34(4):518-21.

13. XARÁ S, et al. Abordagem Nutricional no Doente com Quilotórax. Revista Nutrícias. 2013;(19):32-4.

14. SHARMA AK, et al. Bilateral chylothorax following reoperative central neck dissection for metastatic papillary thyroid cancer. BMJ Case Reports, 2018,2018-224916.

15. SUH S, et al. Outcome prediction with the revised American joint committee on cancer staging system and American thyroid association guidelines for thyroid cancer. Endocrine 2017; 58:495-502.

16. BENDER $B$, et al. The changing management of chylothorax in the modern era. European Journal of Cardio-Thoracic Surgery, 2016;49:18-24.

17. HAYASHIBARA N, et al. Efficacy of octreotide against chylothorax following lateral neck dissection for thyroid cancer: A case report. International Journal of Surgery Case Reports, 2016;21:107-110. 